\title{
MODELLING AQUIFER VULNERABILITY TO NITRATES UNDER THE ASSUMPTION OF VARYING SPATIAL SUPPORT OF WATER WELL DISTRIBUTION
}

\author{
ANDREA G. FABBRI ${ }^{1} \&$ ANTONIO PATERA ${ }^{2}$ \\ ${ }^{1}$ Università di Milano-Bicocca, Milan, Italy \\ ${ }^{2}$ Istituto Nazionale di Geofisica e Vulcanologia, Rome, Italy
}

\begin{abstract}
This contribution analyses the spatial support of sampling points used to express the presence or absence of $\mathrm{NO}_{3}^{-}$pollution in the water table. A spatial database constructed for the assessment of ground water vulnerability is re-analysed with a different predictive strategy. In practice, a case study area surrounding the city of Milan in northern Italy becomes an opportunity to point at a very general prediction modelling problem in which the basic direct evidence of a process is obtained only by sampling with point like measurements of nitrate concentration, as the ones from drill holes or water wells. The main questions are: "What is the functional spatial support for the modelling?" and "What happens if different spatial supports are assumed?" The answers to these questions are counterintuitive. Over the area of study of about $2,000 \mathrm{~km}^{2}$, the distribution of 305 water wells delimits a training area in which 133 wells are considered as impacted by nitrate pollution, i.e., direct supporting patterns of the modelling. The remaining 172 wells are considered as non-impacted. In the training area, nine natural and anthropogenic map data are assumed, as indirect supporting patterns of the modelling, to reflect both the potential source of nitrates and the relative ease in which nitrates may migrate in ground water. They cover the entire area of study. A mathematical model is used that computes spatial relationships between the direct and indirect supporting patterns based on empirical likelihood ratios. The relationships are integrated into prediction patterns and, by iterative cross-validations, into target and uncertainty patterns. These are then extended from the training area over the remaining much larger study areas for analysis and visualization. Square neighbourhoods of dimensions $20 \times 20 \mathrm{~m}, 60 \times 60 \mathrm{~m}$, $180 \times 180 \mathrm{~m}$ and $1,020 \times 1,020 \mathrm{~m}$ around the 305 wells are used to delimit four training areas of different sizes. Surprisingly, the smaller spatial support appears as the most reliable.

Keywords: aquifer vulnerability, nitrate pollution, empirical likelihood ratios, spatial support, prediction patterns, uncertainty patterns, prediction-rate curves.
\end{abstract}

\section{INTRODUCTION}

This contribution focuses narrowly on the effect of spatial support of the sampling points used to express the presence or absence of $\mathrm{NO}_{3}{ }^{-}$pollution in the water table. A spatial database constructed for the prediction of ground water vulnerability is reanalysed using different assumptions on the relationships between water well concentrations of nitrate. The study area of about $2,000 \mathrm{~km}^{2}$ is located around the city of Milan, in northern Italy and the corresponding database was studied by Masetti et al. [1] as a refinement of earlier works [2], [3], who thoroughly discussed the study area, its groundwater contamination problems and the database they constructed. Furthermore, those authors considered new analyses with different threshold values of nitrate concentration [4], [5]; the reliability of different vulnerability classification schemes [6]; and compared positive and negative weights for multiclass generalizations [7]. In those works, the weight-of-evidence model (WoE) was applied for vulnerability assessment.

Methodological discussions on some of those works [1]-[4] led to the generous provision of the database by the original authors for complementary modelling applications in a joint contribution [8]. In it, a different modelling framework and analytical strategy were 
preferred: the empirical likelihood ratio (ELR) function and cross-validation for uncertainty assessment of prediction patterns. Use was made of the immediate vicinity of 305 available water wells measuring the concentration of $\mathrm{NO}_{3}{ }^{-}$in $\mathrm{mg} / \mathrm{l}$ in a training area within the area of study. Of the wells, 133 were considered as "impacted" by nitrate pollution.

As a follow up to their analyses, the Milan area database becomes an opportunity to point at a very general prediction modelling problem in which the basic direct evidence of a process is obtained only by sampling a study area with point like measurements of nitrate concentration, as the ones from drill holes or water wells. The main questions are: "What is the functional spatial support for the modelling?" and "What happens if we assume different spatial supports?"

The next section offers a brief summary of the database and its initial purpose. The favourability modelling framework is then discussed along with the proposed strategies for characterizing, visualizing and cross-validating. Experiments follow on the four training areas to assess the effects of varying spatial support. For this, rank-based statistics, prediction-rate curves, prediction, target, uncertainty and combination patterns are obtained to characterize the training areas and their extension over the respective study areas. Concluding remarks follow with considerations on the importance of assuming realistic and functional spatial support for the water well distribution and values.

\section{THE MILAN AREA OF STUDY DATABASE}

Agricultural practices and industrial activities characterize the area of study around Milan in northern Italy, located as shown in Fig. 1. It covers nearly $2,000 \mathrm{~km}^{2}$ and its groundwater system has a complex hydrogeological setting with interaction of three aquifers [2]. The subsoil sediments represent important water resources. The studies by Masetti et al. [1] focused on the vulnerability of an unconfined aquifer that represents the most affected by contaminants from the surface activities. It is termed Traditional Aquifer and consists of

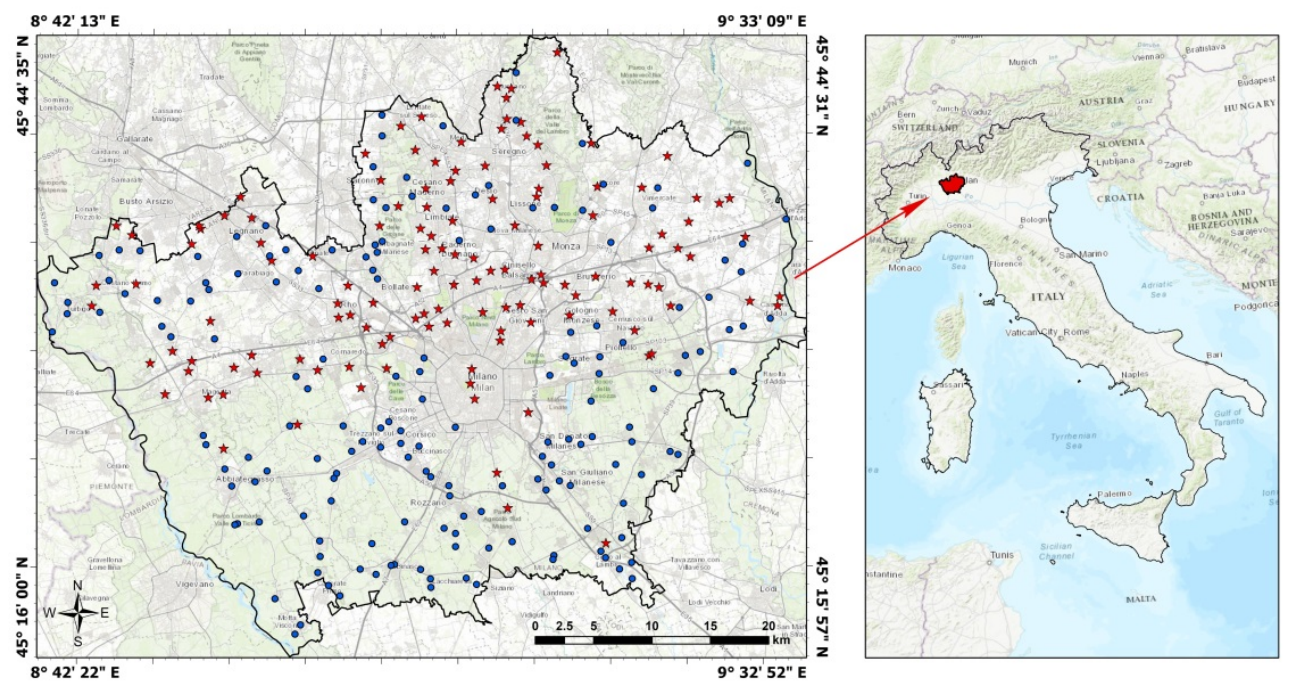

Figure 1: Distribution of 305 water wells in the Milan area of study. Red stars indicate the wells recording $\geq 25 \mathrm{mg} / \mathrm{l}$ of $\mathrm{NO}_{3}{ }^{-}$; blue circles those recording $<25 \mathrm{mg} / \mathrm{l}$. Sparse red stars among cluster of blue circles can be seen, as well as vice versa, indicating a noisy clustered distribution. 
Pliocene-Pleistocene sediments. Transmissivity ranges from $5 \times 10^{-2}$ to $1 \times 10^{-3} \mathrm{~m}^{2} / \mathrm{s}$, with permeability between $5 \times 10^{-3}$ and $1 \times 10^{-8} \mathrm{~m}^{2} / \mathrm{s}$. Thickness ranges from $60-120 \mathrm{~m}$. Components are gravel and sands and clay-silt layers that increase southward. Groundwater depth averages $30 \mathrm{~m}$ to the north of the area reducing to $5 \mathrm{~m}$ to the south.

Information on nitrate concentration was collected from over 300 water wells, unevenly distributed throughout the whole area, as shown in Fig. 1, to monitor four times a year the nitrate concentration that appears not sensitive to seasonality. Concentration varies between $10 \mathrm{mg} / \mathrm{l}$ to the south and $70 \mathrm{mg} / \mathrm{l}$ to the north, with median value around $20 \mathrm{mg} / \mathrm{l}$. The European Community Standard [9] set a guide value in soil of $25 \mathrm{mg} / \mathrm{l}$.

Alberti et al. [2] and Masetti et al. [1] provided a detailed account of the absence of temporal trends and the differences between the northern and the southern parts of the area of study. They employed statistical analyses in their study of regional groundwater vulnerability to spatially relate measured contaminant locations with the distribution of natural and man-induced factor maps. For this, they have constructed a database consisting of impacted and non-impacted water wells selecting the study area and the following natural and anthropogenic factor maps listed in Table 1: groundwater recharge, land use, soil protection capacity, groundwater depth, groundwater velocity, main annual irrigation, nitrogen fertilizer loading, population density and rainfall. Table 1 also lists their short name abbreviations and value ranges.

Table 1: Wells, natural and anthropogenic factors in the Milan study area database (modified after [8]). Note the one-digit short names that will be used to identify the ISPs used for analysis.

\begin{tabular}{|c|c|c|c|}
\hline \multicolumn{4}{|c|}{ Water well data, area of study and direct supporting patterns (DSPs) } \\
\hline Factor map name & Short names & Data range & Description \\
\hline $\begin{array}{l}\text { Impacted wells } \\
\text { Non impacted wells } \\
\text { Area of study }\end{array}$ & $\begin{array}{l}133 \\
172 \\
\text { AS }\end{array}$ & $\begin{array}{l}1-133 \\
1-172 \\
1-0\end{array}$ & $\begin{array}{l}\text { Index } \geq 25 \mathrm{mg} / 1 \mathrm{NO}_{3}{ }^{-} \\
\text {Index } \leq 24 \mathrm{mg} / 1 \mathrm{NO}_{3}{ }^{-} \\
\text {Area and out-of-area } \\
\text { indicator }\end{array}$ \\
\hline \multicolumn{4}{|c|}{ Categorical natural and anthropogenic factors } \\
\hline Factor map name & Short name & Data range & Description \\
\hline $\begin{array}{l}\text { Land use } \\
\text { Soil protection capacity }\end{array}$ & $\begin{array}{l}\text { ldu, L } \\
\text { spc, } S\end{array}$ & $\begin{array}{l}\text { Classes 1-3 } \\
\text { Classes 1-3 }\end{array}$ & $\begin{array}{l}\text { Combination of raf and mai } \\
\times \text { a function of spc as } \\
\text { infiltration coefficient } \\
\text { Urban, agricultural and } \\
\text { woods } \\
\text { Low, moderate and high }\end{array}$ \\
\hline \multicolumn{4}{|c|}{ Continuous natural and anthropogenic factors } \\
\hline Factor map name & Short name & Data range & Description \\
\hline $\begin{array}{l}\text { Ground water depth } \\
\text { Ground water velocity } \\
\text { Main annual irrigation } \\
\text { Nitrogen fertilizer loads } \\
\text { Population density } \\
\text { Rainfall }\end{array}$ & $\begin{array}{l}\text { gwd, d } \\
\text { gwv, , } \\
\text { mai, i } \\
\text { nfl, n } \\
\text { pod, p } \\
\text { raf, r }\end{array}$ & $\begin{array}{l}1-51 \\
112-181 \\
0.1-1531.0 \\
0-428 \\
43-7933 \\
808-1253 \\
\end{array}$ & $\begin{array}{l}\mathrm{m} \\
\text { originally } 10-20-\ln (\mathrm{m} / \mathrm{s}) \\
\mathrm{mm} \\
\mathrm{kg} / \mathrm{h} / \mathrm{y} \\
\text { inhabitants } / \mathrm{km}^{2} \\
\mathrm{~mm} / \mathrm{y}\end{array}$ \\
\hline
\end{tabular}


In essence, the database for the Milan area of study used here consists of a set of 10 digital images contained within a raster of 3,300 pixels by 2,665 lines. Each pixel corresponds to a square of $20 \times 20 \mathrm{~m}$ on the ground. Of the $8,794,500$ pixels in the rectangular raster, only $4,908,305$ cover the area of study, and 3,886,195 are falling out of it. The distribution of the 305 water wells, shown in Fig. 1, is represented as an image with 305 pixels with the values of $\mathrm{NO}_{3}{ }^{-}$, ranging from a maximum of $71.0 \mathrm{mg} / \mathrm{l}$ to a minimum of $10.9 \mathrm{mg} / \mathrm{l}$. Of those, the 133 with value $\geq 25 \mathrm{mg} / 1$ were termed "impacted wells" while the 172 with value $<25 \mathrm{mg} / 1$ were termed "non-impacted wells". Together the sets of impacted and non-impacted wells represent all that is known about nitrate concentration in the groundwater in the area of study so that their location distribution can be used to define training areas for the modelling. Within the training areas, the distribution of sequentially numbered impacted wells is converted into a direct supporting pattern, DSP, and used to establish spatial relationships with the images of natural and anthropogenic factors, converted into indirect supporting patterns, ISP. The relationships are then extended to the remaining study areas.

\section{FAVOURABILITY FUNCTION MODELLING}

The term "favourability function" was proposed [10] to refer to spatial modelling within a unified mathematical framework. Examples of interpretations that were considered are: Bayesian Probability, Certainty Factor, Dempster-Shafer Belief function and Fuzzy Logic. Their implicit assumptions were discussed along with their computations under different database conditions. In particular, integration rules for the models were discussed by Chung and Moon [11]. The ELR model has been thoroughly discussed by Chung [12] and it will be used here.

The modelling with the ELR function generates an image with integrated values ranging from 0 to infinity for each pixel, a prediction image. The array of relative integrated values, however, is difficult to interpret as such, so that a transformation is conveniently made of it into a prediction pattern. In this transformed version all values are ordered from highest to lowest and equal-area ranks are replacing the ratios for each pixel. "Pattern" is to refer to an artificial construct, i.e., a particular way of interpreting and displaying the results of modelling. "Prediction" is implying that it indicates areas in which future occurrences are likely to be found. DSP and ISP have already been defined.

To apply a model, all the known occurrences must be used first, in our case all the 133 impacted sequentially numbered well locations, for instance. This should generate the most informed pattern. However, for interpreting it, the pattern's ability to "predict" future occurrences, i.e. their location or distribution, needs to be studied. We then pretend not to know the location of some of the occurrences ("younger"), apply again the model using the remaining occurrences ("older") as DSP, generate a new prediction pattern and then verify where in it the excluded occurrences are located: hopefully in the higher ranks of the pattern. Of course there are many ways to perform such a cross-validation, as we have termed it. Convenient iterative strategies are of excluding sequentially a few occurrences repeating modelling and cross-validation a number of times. Alternatively, sequential selection or random selection can be preferred. Clearly, the strategy can be tailored to the peculiarities of the available data.

Another critical aspect of spatial prediction modelling is the selection of a training area in which to establish the spatial relationships between DSP and ISP. This is because in it the relationships are considered either more accurate or more easily measurable. From the training area the relationships computed can then be extended to the remaining part of the area of study, termed study area. 
The visual expression of a prediction pattern can be generated by conveniently grouping 200 equal area ranks into fixed and recognizable classes: for instance broader classes for lower ranks of lesser concern and narrower classes for higher ranks. Furthermore, for facilitating comparisons between predictions, the classes must remain the same for all subsequent results of the iterative cross-validations process. Iterations allow computing target patterns, uncertainty patterns and their combination patterns. For instance, using robust statistics such as median and range, we can obtain a target pattern with pixel values of the median rank of the prediction patterns generated by iterations. The values of the uncertainty patterns correspond to the rank of the ranges around the median of the target pattern.

The spatial relationships and their integration by a model do imply assumptions as to the data type available and to the specific mathematical model being used. Examples of data and model assumptions have been discussed in a previous work by Fabbri et al. [8] who used the very same Milan database. They do not need repeating here. We can just point at the following: (i) the assumption that the future occurrences of pollution will take place under conditions similar to the ones represented in the database; and (ii) that the indirect spatial support consists of conditionally independent factor maps. Assumption (i) represents our hope given that we consider as satisfactory, for the past and the future, the information collected in the database. Assumption (ii) is related with the representation and integration rules specific to many mathematical models that were initially formulated for non-spatial factors like medical symptoms for the prescription of medications or identification of diseases. In the geosciences, however, most frequently different thematic maps over the same area are hardly conditionally independent. It becomes of importance then to verify the dependence effects on the modelling.

\section{EXPERIMENTS ON TRAINING AND STUDY AREAS}

We can now formulate assumptions on the spatial support of the water wells: for instance, a pixel area of $20 \times 20 \mathrm{~m}(1 \times 1$ pixels $)$, of $60 \times 60 \mathrm{~m}(3 \times 3$ pixels $)$, of $180 \times 180 \mathrm{~m}(9 \times 9$ pixels $)$ or of $1020 \times 1020 \mathrm{~m}(51 \times 51$ pixels $)$. Having analysed the distribution of the 305 wells and their values, we have found that it is "dispersed", i.e., the average distance of the wells is greater than a hypothetical random distribution. Distances range from $242 \mathrm{~m}$ to 3942 m. The 133 wells are "clustered" and show a hot-spot to the NNE and a cold-spot to the SSW of the area of study, as visible in Fig. 1. There are numerous low-high outliers and a few high-low ones.

However, when gridded into $20 \times 20 \mathrm{~m}$ pixels and used to identify target areas of 305 pixel neighbourhoods containing 133 impacted pixel neighbourhoods, we have to discover what the spatial relationships within the database do contribute to the spatial modelling results as prediction patterns. To do that, four sets of training areas and study areas were computed from the database. They were termed Ta1, Ta3, Ta9 and Ta51, and the respective remaining parts, the areas of study, as Sa1, Sa3, Sa9 and Sa51. The training areas cover, respectively, the following number of pixels of $20 \mathrm{~m}$ resolution: 305, 2,745, 24,706 and 761,161 . The corresponding study areas cover complementary numbers.

The database consists of three types of factor maps: (i) well locations, (ii) categorical natural and anthropogenic factors, and (iii) continuous ones. They are described and short named in Table 1.

For each training area, the modelling applied consists of the steps described in the following sub-sections: (1) calculation of empirical likelihood ratios; (2) generation of prediction patterns later extended to the study areas; (3) computations of iterative cross- 
validations; and (4) generation of target, uncertainty and combination patterns later extended to the study areas

\subsection{Empirical likelihood ratios}

Table 2 lists the empirical likelihood ratio values, ELR, for the nine ISPs corresponding to the different training areas. Values are shown when $>1.5$ for at least one ISP in one or more areas for comparison. In bold fonts are all values $\geq 2$, a tentative value to threshold the ratios. A value of 1 indicates a frequency in the presence of impacted well identical to that in their absence within the training area. A value of 2 indicates a frequency twice that in the absence of an impacted well. The table filters the essential characteristics of likelihood ratio histograms for the three categorical ISPs and ratio functions for the six continuous field ISPs. We can consider the set of ratios as "signatures" of the training areas. The ELR model integrates for each pixel of a training area the empirical likelihood values of the nine ISPs. This generates a prediction image to be transformed into, and interpreted as, a prediction pattern.

Table 2: Empirical likelihood ratios for ISPs are listed for the different training areas. Mostly ratios $>1.5$ are listed and when $\geq \mathbf{2}$ they are in bold. Upper case letters with subscripts indicate the individual categorical map units. Numbers in italics indicate ranges of continuous field values with bracketed maximum value and ratio reached. Note that for spc all ratios are well below 1.5.

\begin{tabular}{|c|c|}
\hline ISP Ta & ELRs $(\geq 2$ and $>1.5)$ \\
\hline gwr Ta1 & $\mathrm{R}_{8} 1.78, \mathbf{R} 93.20$ \\
\hline ldu & $\mathrm{L}_{2} 1.29$ \\
\hline spc & $<1.50, \mathrm{~S}_{1}, \mathrm{~S}_{2}, \mathrm{~S}_{3}$ \\
\hline gwd & $\geq 221.65-24.55(23.15 \max 2.43) ; 30.05-48.50$ (39.95 $\max 4 \mathbf{4 . 0 3})$ \\
\hline gWv *10 & $\geq 2120.59-127.40(125.88 \max \mathbf{2 . 1 0}) ; 163.02-164.00(164.00 \max \mathbf{5 . 6 0})$; \\
\hline mai $* 10$ & $\begin{array}{l}\geq 10.00-1424.74(1.00 \max 1.69) ; \geq 26430.80-11528.62 \text { (11298.99 } \max \\
\text { 2.28); }\end{array}$ \\
\hline $\mathbf{n f l} \div 10$ & $\geq 1684.80-1264.62(894.91 \max 1.58) ; \geq 22166.82-2235.80(2199.93$ \\
\hline nII $=10$ & $\max 2.12) ; 2519.98-2602.65(2553.09 \max 2.16)$ \\
\hline pod & $\geq 22178.94-2546.76$ (2320.91 $\max 2.17) ; 2946.83-5734.54$ (4934.35 \\
\hline & $\max$ 322.85); 6244.34-6496.01 (6496.01 $\max \mathbf{3 . 0 5})$ \\
\hline raf & $\geq 21057.81-1128.09(1100.14 \max \mathbf{4 . 9 3})$ \\
\hline gwr Ta3 & $\mathrm{R}_{8} 1.84, \mathbf{R 9} 3.04$ \\
\hline ldu & $\mathrm{L}_{2} 1.51$ \\
\hline spc & $<1.50, \mathrm{~S}_{1}, \mathrm{~S}_{2}, \mathrm{~S}_{3}$ \\
\hline gwd & $\geq 221.75-24.45(23.10 \max 2.35) ; 30.40-48.40(40.15 \max 4.10)$ \\
\hline gWv $* 10$ & $\geq 1105.21-133.26(117.45 \max 1.60)$ \\
\hline mai $* 10$ & $\geq 10.00-1424.74(1.00 \max 1.69) ; \geq 26430.80-11528.62(11222.45$ \\
\hline $\mathbf{n f l} * 10$ & $\max 2.29)$ \\
\hline pod & $\geq 12127.17-2931.82(2461.01 \max 1.63)$ \\
\hline f & $\geq 23054.22-5688.01$ (4942.29 $\max 35.10) ; 6251.26-7933.08$ (7933.08 \\
\hline raf & $\begin{array}{l}\max \text { 7030.60); } \\
\geq \mathbf{2} 1086.14-1129.78(1107.34 \max \mathbf{2 . 1 6})\end{array}$ \\
\hline
\end{tabular}


Table 2: Continued.

\begin{tabular}{|c|c|}
\hline ISP Ta & $\operatorname{ELRs}(\geq \mathbf{2}$ and $>1.5)$ \\
\hline $\begin{array}{l}\text { gwr Ta9 } \\
\text { ldu } \\
\text { spc } \\
\text { gwd } \\
\text { gwv } * 10 \\
\text { mai } * 10 \\
\text { nfl } * 10 \\
\text { pod } \\
\text { raf }\end{array}$ & $\begin{array}{l}\mathrm{R}_{8} 1.75, \mathbf{R} \mathbf{3 . 1 7} \\
\mathbf{L}_{2} \mathbf{2 . 0 9} ; \\
<1.50, \mathrm{~S}_{1}, \mathrm{~S}_{2}, \mathrm{~S}_{3} ; \\
\geq 221.80-24.25(24.05 \max \mathbf{2 . 2 9}) ; 30.05-48.10(39.95 \max \mathbf{4 . 1 3}) ; \\
\geq 1105.06-133.27(117.44 \max 1.59) ; \\
\geq 10.00-1423.83(15.31 \max 1.67) ; \geq \mathbf{2} 6430.22-11528.36(7838.75 \\
\max \mathbf{2 . 3 0}) ; \\
\geq 12127.17-2931.82(2461.01 \max 1.63) ; \\
\geq 23078.02-5688.01(4942.29 \max \mathbf{3 4 . 8 9}) ; 6251.26-7933.08(7933.08 \\
\max \mathbf{7 0 3 2 . 3 9}) ; \\
\geq 21085.75-1129.44(1106.97 \max \mathbf{2 . 1 5}) .\end{array}$ \\
\hline $\begin{array}{l}\text { gwr Ta51 } \\
\text { ldu } \\
\text { spc } \\
\text { gwd } \\
\text { gwv } * 10 \\
\text { mai } * 10\end{array}$ & $\begin{array}{l}\mathrm{R}_{8} 1.62, \mathbf{R} \mathbf{3 . 3 8} ; \\
\mathrm{L}_{2} 1.34 ; \\
<1.50, \mathrm{~S}_{1}, \mathrm{~S}_{2}, \mathrm{~S}_{3} ; \\
\geq 221.35-23.90(22.55 \max \mathbf{2 . 1 6}) ; 29.85-45.55(38.25 \max \mathbf{3 . 6 9}) ; \\
\geq 11.00-57.88(\max 1.70) ; 102.34-133.30(116.92 \max 1.57) ; \\
\geq 10.00-1224.00(229.65 \max 1.62) ; 6169.95-6445.53 ; \geq 26460.84- \\
11513.05(7348.82 \max \mathbf{2 . 2 0}) ; \\
\geq 12110.05-2863.34(2362.57 \max 1.82) ; \\
\geq 23268.41-5703.87(4942.29 \max \mathbf{4 1 . 0 6}) ; 6338.52-7903.08(7903.08 \\
\max \mathbf{4 8 8 1 . 5 1}) ; \\
\geq 10.00-411.58(248.95 \max 1.70) ; \geq 21085.86-1129.64(1107.13 \max \\
\mathbf{2 . 1 6}) .\end{array}$ \\
\hline
\end{tabular}

Comparing the ratios for the four training areas we can observe the following similarity and differences: (1) $\mathbf{R}_{\mathbf{9}}$ is high, $>3$ in all areas; (2) $\mathbf{L}_{2}$ is $>2$ only for Ta9; (3) spc is low, $<1.5$ in all areas; (4) gwd, mai, pod and raf are $>2$ in all areas, however, raf is higher, 4.93 in Ta1; and (5) gwv is $>2$ only in Ta1.

These differences in ELR values appear as minor and we may expect similar modelling results. The likelihood values are characteristic properties of the spatial database, measuring the spatial relationships between DSP and ISPs in the training areas. We can consider the ratios in Table 2 as database signatures. The mathematical modelling, in our case by the ELR function model, will integrate the ratios for each point or pixel in the database, under a number of assumptions and following specific combination rules. Recall that the ratios range in values between zero and infinity and that the values are difficult to interpret. Having the signatures of the training areas we can now try to see their effects on the modelling of the respective prediction patterns.

\subsection{Prediction patterns}

ELR prediction patterns were obtained for the four training areas: ELR_Ta1_133_RLS_dvinpr to ELR_Ta51_133_RLS_dvinpr. They are named using the abbreviation of the mathematical model, ELR, followed by the identification of the training 
area, the DSP of the impacted wells, and the list of categorical and continuous ISPs, as upper case and lower case abbreviations, respectively.

Because the images of the training areas consist of groups of single pixels or of small pixel neighbourhoods, their colour display is not informative, except for Ta51 where the neighbourhoods are larger, as done in Fig. 4(a) and 4(b). However, when using the modelling statistics from the training areas to extend it to the corresponding study areas, different prediction patterns are generated as follows ( $\mathbf{X}$ indicates extension, and $\mathbf{S a}$ the study areas): XLR_Sa1_133_RLS_dvinpr to XLR_Sa51_133_RLS_dvinpr. They are displayed in Fig. 2. There we can see the similarity of selected ranks in the patterns and also some minor differences of particular relevance for the $10 \%$ highest classes of ranks. Note that the legend's ranked classes are wider for lower ranks and narrower for higher ranks. The pseudo-colouring scheme goes from cold to warm colours but the class boundaries remain fixed in order to facilitate recognition and allow comparison.

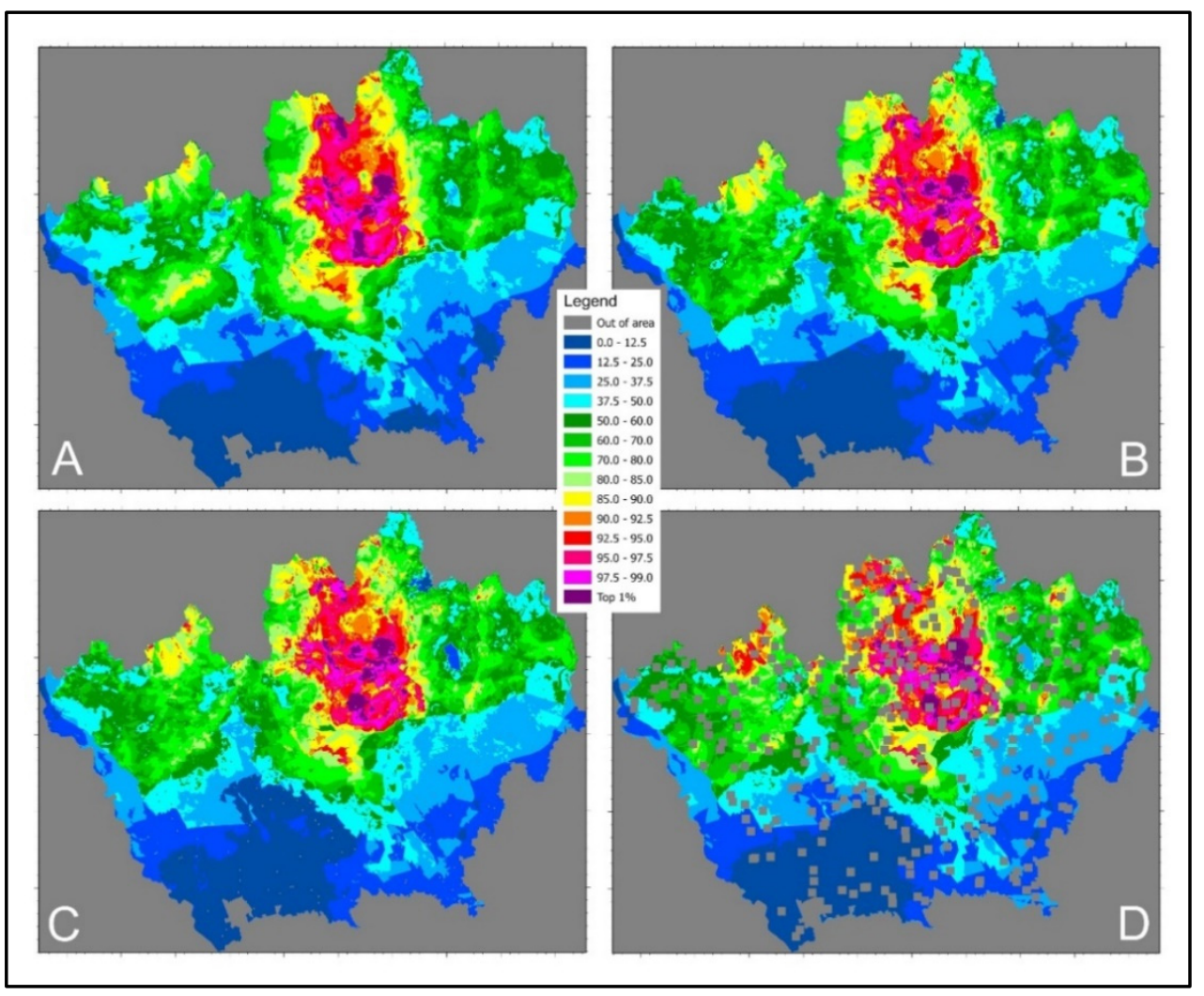

Figure 2: $\quad$ Prediction patterns for study areas. XLR prediction pattern for Sa1 are in (A); for Sa3 in (B), for Sa9 in (C); and for Sa51 (D). Explanation is in text.

We can observe the following characteristics in Fig. 2: (1) greater compactness of colours in the prediction pattern in Fig. 2(a); (2) strong similarities of higher classes for the top 1\% (purple) and top 5\% (red to purple); (3) patches of high value colours to the west and the east 
in Fig. 2(b), 2(c) and 2(d) (yellow to red); (4) altogether rather similar prediction patterns for the four study areas with a large hot area to the NNE of the city of Milan, the industrial zone.

At this point it becomes instructive to ask: How good are the prediction patterns as predictor of areas of future impacted wells? How stable and how certain? Clearly it has to depend on how good predictors are the patterns generated from the respective training areas, Ta1 to Ta51. So far we can only consider fitting rates of the impacted wells within the ranks generated using them as DSP. They do not provide any information on predictive capability. To obtain some measure of effectiveness in predicting we can use strategies of blind testing via iterative cross-validation of prediction patterns.

\subsection{Iterative cross-validations}

A number of strategies can be formulated all based on pretending to ignore some of the sample points available. In our case they are the 133 impacted wells (pixels or pixel neighbourhoods) and are critical for establishing the spatial relationships between DSP and ISPs. First we use all the 133 wells to generate the best or most informed prediction patterns, as shown in Fig. 2. Then we repeat the analyses by pretending not to know some relevant numbers of impacted wells.

For instance, in case of only a few tens of wells available, we can sequentially exclude one and use the remaining n-1 for modelling new prediction patterns. Then we validate them with the prediction rates corresponding to the excluded well locations. We can iterate the process $\mathrm{n}$ times to obtain $\mathrm{n}$ prediction rates, one per excluded impacted well. The distribution of the rates as ranks throughout those of the prediction pattern is obtained as a table and a corresponding prediction-rate histogram or cumulative curve. In Fig. 3, for instance, we have used the strategy of excluding 8 wells from the 133, using the remaining 125 for modelling in the iterative cross-validation process. This generates 16 prediction patterns each validated by 8 prediction rates. Another strategy used was of selecting at random 93 wells out of the 133 (about the 70\%) and repeating the modelling 16 times for the four training areas. That did generate results very similar to the ones in Fig. 3.

In the illustration the prediction-rate curves were calculated for the four training areas, Ta1 to Ta51. The diagram in Fig. 3(a) shows the relative proportion of training areas ranked as vulnerable in decreasing order on the horizontal axis and the corresponding cumulative proportion of impacted wells in the class on the vertical axis. Immediately it can be seen that the Ta1 prediction-rate curve is very steep. For Ta3 to Ta51 the curves are increasingly shallower. In addition, the histograms in Fig. 3(b), that consider just the top 20\% ranks in classes of $4 \%$ of training areas, show a monotonically increasing histogram for Ta1, red columns, representing an acceptable classification of vulnerable areas. The blue columns, instead, show for Ta3 a non-increasing histogram, as well as for the corresponding histograms for Ta9 and Ta51, not shown here.

We can observe that the $100 \%$ of impacted wells (vertical axis) are ranked within the top $2 \%$ for Ta1, the top $19 \%$ for Ta3, the top $82 \%$ for Ta9 and the top $85 \%$ for Ta51 (horizontal axis). The histogram for Ta1, in Fig. 3(b), indicates a good classification, with the higher equal area classes in $4 \%$ intervals and monotonically increasing columns towards higher ranks.

Which curve is the one representing a better prediction pattern? Is that because it contains more impacted wells at higher ranks? Or is it because the prediction pattern has less uncertainty associated? 

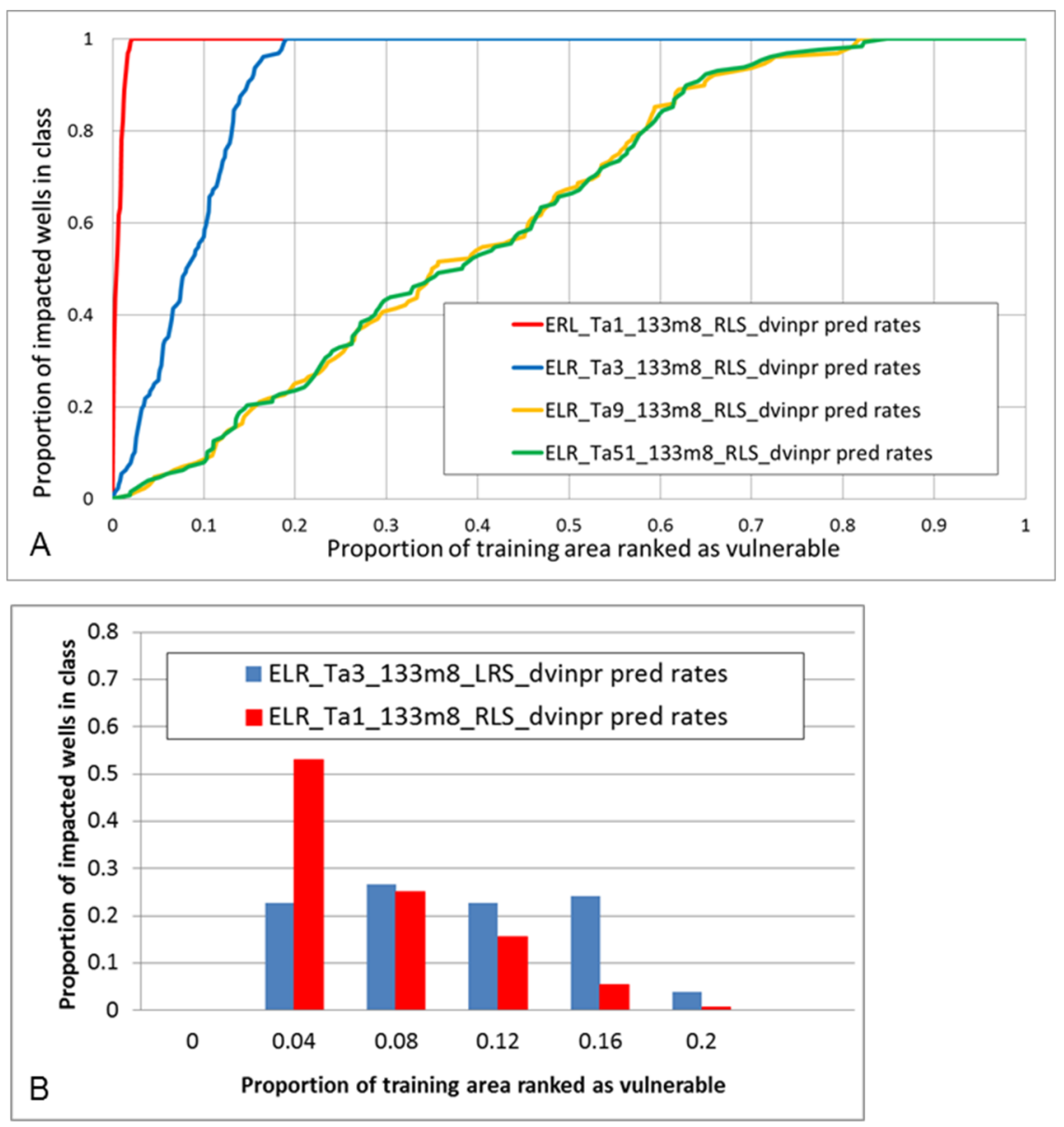

Figure 3: ELR prediction-rate curves and histograms. In (A) for the four training areas Ta1 to Ta51, obtained using the iterative cross-validation strategy of sequential exclusion of eight impacted wells out of 133. The process generates 16 prediction patterns each cross-validated by eight excluded wells. The curves for Ta9 and Ta51 reflect nearly random distribution of ranks. In (B) are the histograms for Ta1, monotonically increasing, and Ta3, non-increasing.

\subsection{Target and uncertainty patterns}

To answer this type of question we can proceed to generate the 16 prediction patterns out of the 133 minus $8 \times 16$ strategies for the four training areas to generate target and uncertainty patterns as done in Fig. 4(a) and 4(b) for Ta51. In Fig. 4(c) and 4(d) for Sa51 we have extended the statistics obtained from Ta51 to Sa51. The target patterns in the illustration have been obtained from the set of 16 prediction patterns for Ta51. The median of the 16 ranks (of the 16 patterns) is selected for each pixel and becomes the rank of the target pattern, as shown in Fig. 4(a) and 4(c). 


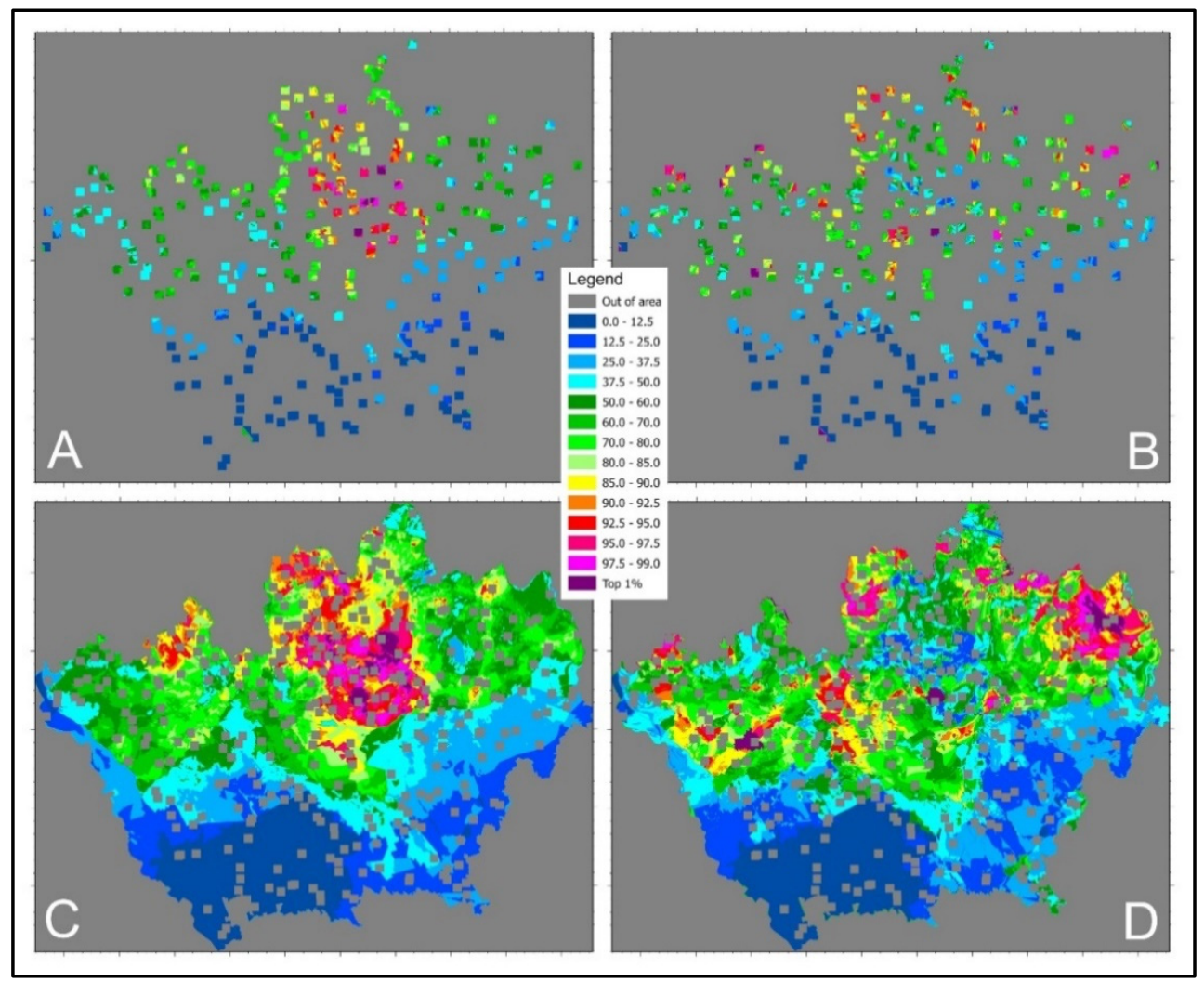

Figure 4: Target and uncertainty patterns for training and study areas. ELR target pattern for Ta51 is in (A); uncertainty pattern for Ta51 in (B), XLR target pattern for Sa51 in (C); and uncertainty pattern for Sa51 in (D). Explanation is in text.

Visually, the target pattern is very similar to the prediction pattern (compare Fig. 4(c) and Fig. 2(d)). However, the range of the 16 ranks represents an estimation of the uncertainty in the ranking. The wider is the range the more uncertain can the target patterns be considered and consequently also the initial prediction pattern. The same legend is being used for the illustrations of target and uncertainty patterns in Fig. 4. Obviously, the significance of the uncertainty ranks, in Fig. 4(b) and 4(d), is the reverse the one of the target ranks, in Fig. 4(a) and 4(c) For instance, we have selected the $50 \%$ lowest values from the uncertainty pattern to identify all the ranks in the target pattern (or the prediction pattern) corresponding to lower uncertainty. This produced the combination patterns shown in Fig. 5. Observe in Fig. 5(d) the 50\% combination pattern for Sa51, obtained combining the patterns in Fig. 4(d), uncertainty, with the one in Fig. 4(c), target pattern. We have applied median and range statistics here, due to its robustness, differently from the previous work [8] where the more sensitive mean and variance were tentatively used.

Evaluating and comparing uncertainty patterns is a complex issue, worthy of extensive research. The four $50 \%$ combination patterns in Fig. 5 show strong differences in the distribution of the ranks. All our modelling is based on relative measures and on ranking 


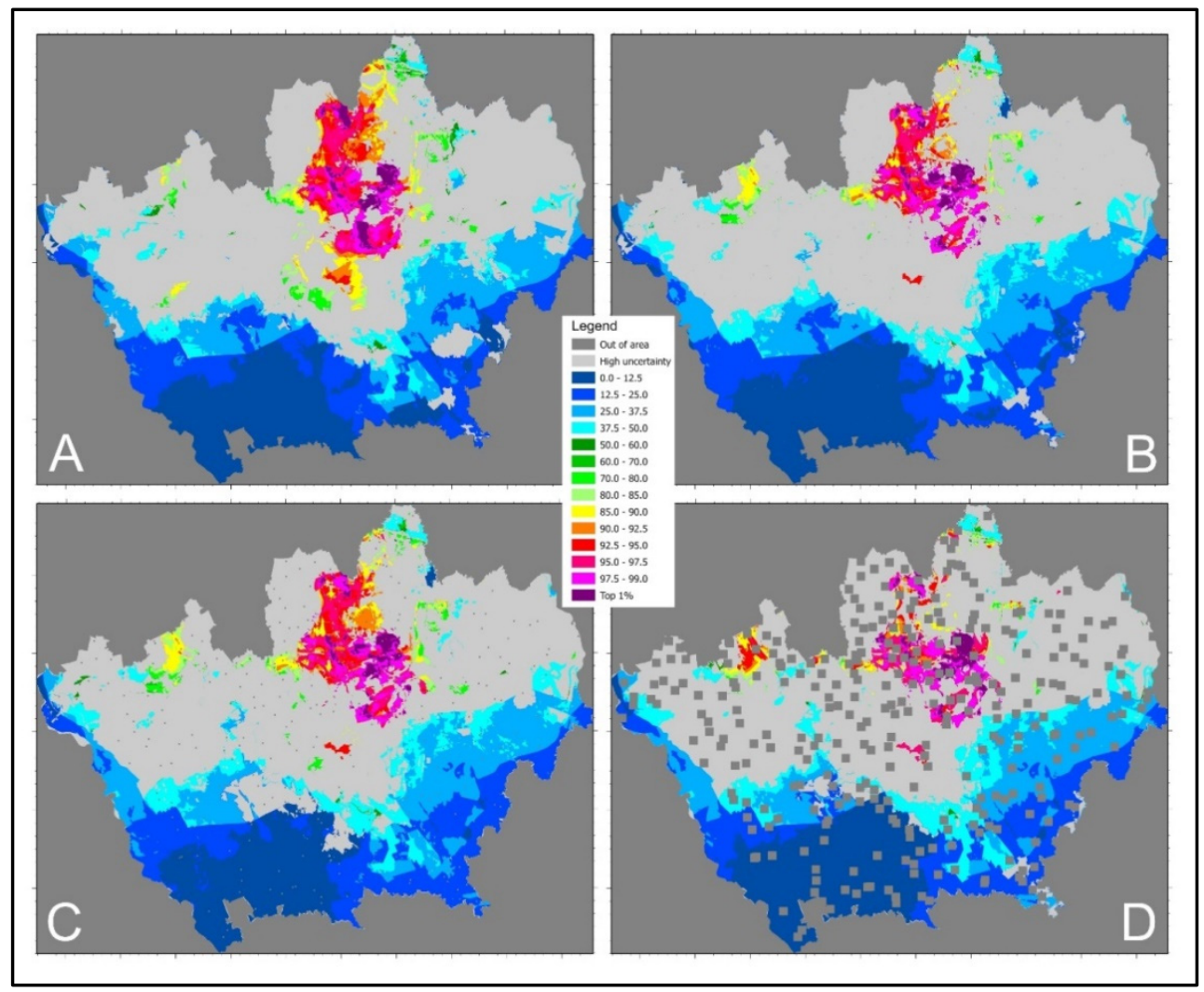

Figure 5: XLR 50\% combination patterns of uncertainty and target patterns for four study areas. In (A) is for Sa1, in (B) for Sa3, in (C) for Sa9 and in (D) for Sa51. Explanation is in text.

statistics. What we could do in a rough empirical manner is to compare ranks for a given top set or class. For instance, by arbitrarily selecting the top $10 \%$ ranks, we can evaluate the loss of the top target rank area in the 50\% combination patterns. They mask the part of the target patterns with higher uncertain ranks (belonging to the higher $50 \%$ of the uncertainty pattern). For the patterns in Fig. 5(a)-5(d), we have a relative decrease for the top 10\% target ranks as follows: of $29.64 \%$, for Sa1, of $46.54 \%$ for $\mathbf{S a 3}$, of $39.88 \%$ for Sa9, and finally of $61.33 \%$ for Sa51. This reveals a relatively greater uncertainty affecting Ta3 to Ta51 than that affecting Ta1. In other words, it indicates a better ranking for Ta1 with less relative uncertainty.

\section{CONCLUSIONS}

This contribution reanalyses a database constructed for assessing aquifer vulnerability to nitrate pollution around the city of Milan. The effects of spatial support are explored and a narrow support appears to generate more robust and less uncertain vulnerability ranks. Favourability function modelling with the empirical likelihood ratio is applied to four training areas extracted from the database. The areas simulate increasingly wider spatial support of water well values of nitrate concentration. The statistics resulting from the 
prediction patterns and their cross-validations is extended from the training areas to the surrounding study areas. In this way the uncertainty patterns are computed and compared using rank and range statistics. This is done in parallel with the predictive capability of the prediction patterns represented by prediction-rate curves and histograms.

Increasing the extension of training areas leads to prediction patterns that, in spite of their similarity, imply the worsening of prediction quality, the loss of monotonically increasing character of ranking, and an increase of the uncertainty associated with the target and prediction patterns. The $20 \mathrm{~m}$ neighbourhood training area appears preferable to the wider ones. The assumptions of broader spatial supports generate classifications that are degrading with the broadening of the support. Such aspects must be considered when applying prediction models to areas for which the vulnerable occurrences, i.e., the impacted wells, consist of point values sampling a process. The training areas being modelled have to be limited operational neighbourhoods of the points, water wells, because their distribution, spacing and variability are visibly affecting the resulting prediction patterns. The many implications of the results obtained from the Milan area of study make it worth further attention. More generally, research issues worthy of consideration and linked to the assessment of spatial support, are: comparisons of prediction patterns and prediction-rate curves, and comparisons of relative uncertainty of target patterns.

\section{REFERENCES}

[1] Masetti, M., Poli, S. \& Sterlacchini, S., The use of weights-of-evidence modeling technique to estimate the vulnerability of groundwater to nitrate contamination. Natural Resources Research, 16(2), pp. 109-119, 2007.

[2] Alberti, L., DeAmicis, M., Masetti, M. \& Sterlacchini, S., Bayes rule and GIS for evaluating sensitivity of groundwater contamination. Proceedings of the IAMG 2001 Conference, 6-12 Sep., Cancun, Mexico, 18 pp., 2001. www.iamg.org.

[3] Masetti, M., Poli, S. \& Sterlacchini, S., Aquifer vulnerability assessment using weights of evidence modelling technique: Application to the Province of Milan, northern Italy. Proceedings of the IAMG 2005 Conference: GIS and Spatial Analysis, 21-25 Aug., Toronto, Canada, 1, pp. 499-504, 2005.

[4] Masetti, M., Sterlacchini, S., Ballabio, C., Sorichetta, A. \& Poli, S., Influence of threshold value in the use of statistical methods for groundwater vulnerability assessment. Science for the Total Environment, 407, pp. 3836-3846, 2009.

[5] Masetti, M., Sorichetta, A., Ballabio, C., Sterlacchini, S. \& Pozzi, M., Using different thresholds in assessing ground-water vulnerability through statistical methods. Proceedings of the IAMG 2011 Conference, 5-9 Sep., Salzburg, Austria, pp. 959-969, 2011.

[6] Sorichetta, A., Masetti, M., Ballabio, C., Sterlacchini, S. \& Beretta, G.P., Reliability of groundwater vulnerability maps obtained through statistical methods. Journal of Environmental Management, 92, pp. 1215-1224, 2011.

[7] Sorichetta, A., Masetti, M., Ballabio, C. \& Sterlacchini, S., Aquifer nitrate vulnerability assessment using positive and negative weights of evidence methods, Milan, Italy. Computers and Geosciences, 48, pp. 199-210, 2012.

[8] Fabbri, A.G., Cavallin, A., Masetti, M., Poli, S., Sterlacchini, S. \& Chung, C.-J., Spatial uncertainty of groundwater-vulnerability predictions assessed by a crossvalidation strategy: An application to nitrate concentrations in the Province of Milan, northern Italy. Risk Analysis VII and Brownfields V, ed. C.A. Brebbia, WIT Press: Southampton and Boston, pp. 497-514, 2010. 
[9] European Community, Council Directive 91/676/EEC of 12 Dec. 1991 concerning the protection of waters against pollution caused by nitrates from agricultural sources, (Nitrate Directive) OJ L 375, 31.12.1991, pp. 1-8, 1991.

[10] Chung, C.F. \& Fabbri, A.G., The representation of geoscience information for data integration. Nonrenewable Resources, 2(2), pp. 122-139, 1993.

[11] Chung, C.F. \& Moon, W.M., Combination rules of spatial geoscience data for mineral exploration. Geoinformatics, 2, pp. 159-169, 1991.

[12] Chung, C.F., Using likelihood ratio functions for modelling the conditional probability of occurrence of future landslides for risk assessment. Computers and Geosciences, 32, pp. 1025-1065, 2006. 